Bull. Korean Math. Soc. 50 (2013), No. 6, pp. 1841-1846

http://dx.doi.org/10.4134/BKMS.2013.50.6.1841

\title{
MORE ON SUMS OF HILBERT SPACE FRAMES
}

\author{
A. Najati, M. R. Abdollahpour, E. Osgooei, and M. M. Saem
}

ABSTRACT. In this paper we establish some new results on sums of Hilbert space frames and Riesz bases. We also provide a correction to some recently established results in [2].

\section{Introduction}

Throughout this paper, $\mathcal{H}$ denotes a separable Hilbert space with the inner product $\langle\cdot, \cdot\rangle$. Recall that a sequence $\left\{f_{i}\right\}_{i \in I} \subseteq \mathcal{H}$ is a frame for $\mathcal{H}$ if there exist $0<A \leqslant B<\infty$ such that

$$
A\|f\|^{2} \leqslant \sum_{i \in I}\left|\left\langle f, f_{i}\right\rangle\right|^{2} \leqslant B\|f\|^{2}
$$

for all $f \in \mathcal{H}$. The constants $A$ and $B$ are called a lower and upper frame bounds, respectively.

We call a sequence $\left\{f_{i}\right\}_{i \in I} \subseteq \mathcal{H}$ a Bessel sequence for $\mathcal{H}$, if the right hand inequality in (1.1) holds for all $f \in \mathcal{H}$.

Let $\left\{f_{i}\right\}_{i \in I}$ be a Bessel sequence for $\mathcal{H}$. Then the bounded operator

$$
T: \mathcal{H} \rightarrow l_{2}, \quad T f=\left\{\left\langle f, f_{i}\right\rangle\right\}_{i \in I}
$$

is called the analysis operator of $\left\{f_{i}\right\}_{i \in I}$ and its adjoint

$$
T^{*}: l_{2} \rightarrow \mathcal{H}, \quad T^{*}\left(\left\{c_{i}\right\}_{i \in I}\right)=\sum_{i \in I} c_{i} f_{i},
$$

is called the synthesis operator of $\left\{f_{i}\right\}_{i \in I}$. If $\left\{f_{i}\right\}_{i \in I}$ is a frame for $\mathcal{H}$, the frame operator for $\left\{f_{i}\right\}_{i \in I}$ is the operator $S: \mathcal{H} \rightarrow \mathcal{H}$ given by $S f=\sum_{i \in I}\left\langle f, f_{i}\right\rangle f_{i}$. It is clear that $\langle S f, f\rangle=\sum_{i \in I}\left|\left\langle f, f_{i}\right\rangle\right|^{2}$ for all $f \in \mathcal{H}$. Therefore, $S$ is positive and invertible. This provides the frame decomposition

$$
f=\sum_{i \in I}\left\langle f, S^{-1} f_{i}\right\rangle f_{i}=\sum_{i \in I}\left\langle f, f_{i}\right\rangle S^{-1} f_{i}
$$

for all $f \in \mathcal{H}$.

Received February 18, 2012; Revised July 29, 2013.

2010 Mathematics Subject Classification. Primary 41A58, 42C15.

Key words and phrases. frame, Gabor frame, frame operator. 
A sequence $\left\{f_{i}\right\}_{i \in I} \subseteq \mathcal{H}$ is called a Riesz basis for $\mathcal{H}$, if $\overline{\operatorname{span}}\left\{f_{i}\right\}_{i \in I}=\mathcal{H}$ and there exist $0<A \leqslant B<\infty$ such that

$$
A \sum_{i}\left|c_{i}\right|^{2} \leqslant\left\|\sum_{i} c_{i} f_{i}\right\|^{2} \leqslant B \sum_{i}\left|c_{i}\right|^{2}
$$

holds for every finite scalar sequence $\left\{c_{i}\right\}$. The constants $A$ and $B$ are called the lower and upper Riesz basis bounds, respectively.

We will use the following lemma in the rest of paper.

Lemma 1.1 ([1], Lemma A.7.1). If $\mathcal{H}$ and $\mathcal{K}$ are Hilbert spaces and $T: \mathcal{H} \rightarrow \mathcal{K}$ is a bounded operator with closed range, then there exists a bounded operator $T^{\dagger}: \mathcal{K} \rightarrow \mathcal{H}$ such that

$$
T T^{\dagger} T f=T f, \quad f \in \mathcal{H} .
$$

The operator $T^{\dagger}$ is called a pseudo-inverse of $T$.

\section{Main results}

The following assertion is stated in [2] as Proposition 2.1.

Assertion 2.1. Let $\left\{f_{i}\right\}_{i \in I}$ be a frame for $\mathcal{H}$ with the frame operator $S$, frame bounds $A \leqslant B$ and let $L: \mathcal{H} \longrightarrow \mathcal{H}$ be a bounded operator. Then $\left\{L f_{i}\right\}_{i \in I}$ is a frame for $\mathcal{H}$ if and only if $L$ is invertible on $\mathcal{H}$. Moreover, in this case the frame operator for $\left\{L f_{i}\right\}_{i \in I}$ is $L S L^{*}$ and the new frame bounds are $A\left\|L^{-1}\right\|^{-2}, B\|L\|^{2}$.

In this note, we show that Assertion 2.1 is not true in general. Indeed, if $\left\{f_{i}\right\}_{i \in I}$ is a frame for Hilbert space $\mathcal{H}$ and $L: \mathcal{H} \longrightarrow \mathcal{H}$ is a bounded invertible operator, then $\left\{L f_{i}\right\}_{i \in I}$ is a frame for $\mathcal{H}$ but the converse is not true in general. In the proof of Proposition 2.1 of [2], the authors proved that $L S L^{*}$ is invertible. But, this does not imply that $L$ is invertible on $\mathcal{H}$. It should be noted that in [2], Proposition 2.1 has been used in Corollaries 2.2, 2.3 and in the proof of Proposition 4.1.

Example 2.2. Let $\left\{e_{n}\right\}_{n=1}^{\infty}$ be an orthonormal basis for a Hilbert space $\mathcal{H}$. Define a shift operator $L$ on $\mathcal{H}$ by $L\left(e_{n}\right)=e_{n-1}$ if $n>1$ and $L\left(e_{1}\right)=0$. It is clear that $\left\{L\left(e_{n}\right)\right\}_{n=1}^{\infty}$ is a frame for $\mathcal{H}$, but $L$ is not invertible although $L L^{*}=I$. Moreover, $\left\{L^{*}\left(e_{n}\right)\right\}_{n=1}^{\infty}$ is not a frame for $\mathcal{H}$.

We can correct Assertion 2.1 as follows:

Proposition 2.3. Let $\left\{f_{i}\right\}_{i \in I}$ be a frame for $\mathcal{H}$ with the frame operator $S$, frame bounds $A \leqslant B$ and let $L: \mathcal{H} \longrightarrow \mathcal{H}$ be a bounded operator. Then $\left\{L f_{i}\right\}_{i \in I}$ is a frame for $\mathcal{H}$ if and only if $L$ is surjective. Moreover, in this case the frame operator for $\left\{L f_{i}\right\}_{i \in I}$ is $L S L^{*}$ and the new frame bounds are $A\left\|L^{\dagger}\right\|^{-2}$ and $B\|L\|^{2}$, where $L^{\dagger}$ is the pseudo-inverse of $L$.

Proof. If $\left\{L f_{i}\right\}_{i \in I}$ is a frame for $\mathcal{H}$, then its frame operator $L S L^{*}$ is invertible. So $L$ is surjective. The converse follows from Corollary 5.3.2 of [1]. 
We also have:

Proposition 2.4. Let $\left\{f_{i}\right\}_{i \in I}$ be a frame for $\mathcal{H}$ with the frame operator $S$ and let $L: \mathcal{H} \longrightarrow \mathcal{H}$ be a bounded operator. Then $\left\{L f_{i}\right\}_{i \in I}$ and $\left\{L^{*} f_{i}\right\}_{i \in I}$ are frames for $\mathcal{H}$ if and only if $L$ is invertible. Moreover, in this case the frame operators for $\left\{L f_{i}\right\}_{i \in I}$ and $\left\{L^{*} f_{i}\right\}_{i \in I}$ are $L S L^{*}$ and $L^{*} S L$, respectively.

Proof. If $\left\{L f_{i}\right\}_{i \in I}$ and $\left\{L^{*} f_{i}\right\}_{i \in I}$ are frames for $\mathcal{H}$, then their frame operators $L S L^{*}$ and $L^{*} S L$ are invertible. So $L$ and $L^{*}$ are surjective and $L$ is invertible. The converse is clear.

In [2], Corollary 2.2 can be corrected as below.

Corollary 2.5. Let $\left\{f_{i}\right\}_{i \in I}$ be a frame for $\mathcal{H}$ with the frame operator $S$, frame bounds $A \leqslant B$ and let $L: \mathcal{H} \longrightarrow \mathcal{H}$ be a bounded operator. Then $\left\{f_{i}+L f_{i}\right\}_{i \in I}$ is a frame for $\mathcal{H}$ if and only if $I+L$ is surjective. Moreover, in this case the frame operator for the new frame is $(I+L) S\left(I+L^{*}\right)$ with the frame bounds $A\left\|(I+L)^{\dagger}\right\|^{-2}$ and $B\|I+L\|^{2}$, where $(I+L)^{\dagger}$ is a pseudo-inverse of $I+L$. In particular, if $L$ is a positive operator (or just $L>-I$ ), then $\left\{f_{i}+L f_{i}\right\}_{i \in I}$ is a frame for $\mathcal{H}$ with the frame operator $S+S L+S L^{*}+L S L^{*}$.

Corollary 2.6. Let $\left\{f_{i}\right\}_{i \in I}$ be a frame for $\mathcal{H}$ and $P: \mathcal{H} \longrightarrow \mathcal{H}$ be a bounded operator. If $P^{2}=P$, then for all $a \neq-1,\left\{f_{i}+a P f_{i}\right\}_{i \in I}$ is a frame for $\mathcal{H}$.

Proof. If $a \neq-1$, then we have $(I+a P)\left(I-\frac{a}{a+1} P\right)=I$. This implies that $I+a P$ is invertible and so $\left\{f_{i}+a P f_{i}\right\}_{i \in I}$ is a frame for $\mathcal{H}$.

Proposition 2.7. Let $\left\{f_{i}\right\}_{i \in I}$ be a sequence in $\mathcal{H}$ such that $\sum_{i \in I}\left\langle f, f_{i}\right\rangle f_{i}$ converges for all $f \in \mathcal{H}$. If $L: \mathcal{H} \longrightarrow \mathcal{H}$ is a bounded operator such that $\left\{L f_{i}\right\}_{i \in I}$ and $\left\{L^{*} f_{i}\right\}_{i \in I}$ are frames for $\mathcal{H}$, then $\left\{f_{i}\right\}_{i \in I}$ is a frame for $\mathcal{H}$.

Proof. Let us define

$$
U: \mathcal{H} \longrightarrow \mathcal{H}, \quad U(f):=\sum_{i \in I}\left\langle f, f_{i}\right\rangle f_{i} .
$$

Let $S_{L}$ be the frame operator for $\left\{L f_{i}\right\}_{i \in I}$. Then $S_{L}=L U L^{*}$ is invertible. So $L$ is surjective. Similarly, we infer that $L^{*}$ is surjective. Therefore $L$ is invertible and so $\left\{f_{i}\right\}_{i \in I}$ is a frame for $\mathcal{H}$ with the frame operator $L^{-1} S_{L}\left(L^{*}\right)^{-1}$.

Proposition 2.8. Let $\left\{f_{i}\right\}_{i \in I}$ be a Riesz basis for $\mathcal{H}$ with analysis operator $T$, Riesz basis bounds $A \leq B$, and let $L: \mathcal{H} \longrightarrow \mathcal{H}$ be a bounded operator. Then $\left\{L f_{i}\right\}_{i \in I}$ is a Riesz basis for $\mathcal{H}$ if and only if $L$ is invertible on $\mathcal{H}$. Moreover in this case, the analysis operator for $\left\{L f_{i}\right\}_{i \in I}$ is $T_{L}=T L^{*}$ and the new Riesz basis bounds are $\left\|L^{-1}\right\|^{-2} A,\|L\|^{2} B$.

Proof. Since the analysis operator for $\left\{L f_{i}\right\}_{i \in I}$ is $T_{L}=T L^{*}, L$ is invertible if and only if $\left\{L f_{i}\right\}_{i \in I}$ is a Riesz basis for $\mathcal{H}$. 
Corollary 2.9. If $\left\{f_{i}\right\}_{i \in I}$ is a Riesz basis for $\mathcal{H}$ and $L: \mathcal{H} \longrightarrow \mathcal{H}$ is a bounded operator, then $\left\{f_{i}+L f_{i}\right\}_{i \in I}$ is a Riesz basis for $\mathcal{H}$ if and only if $I+L$ is invertible on $\mathcal{H}$. In this case the analysis operator for new frame is $T_{I+L}=T\left(I+L^{*}\right)$ and the new Riesz basis bounds are $\left\|(I+L)^{-1}\right\|^{-2} A,\|I+L\|^{2} B$.

We recall that if $\left\{f_{i}\right\}_{i \in I}$ is a frame for $\mathcal{H}$, then the frame $\left\{g_{i}\right\}_{i \in I}$ is called an alternate dual frame of $\left\{f_{i}\right\}_{i \in I}$, if

$$
f=\sum_{i \in I}\left\langle f, g_{i}\right\rangle f_{i}, \quad f \in \mathcal{H} .
$$

Corollary 2.10. Let $\left\{f_{i}\right\}_{i \in I}$ be a Riesz basis for $\mathcal{H}$ with frame operator $S$ and $\left\{g_{i}\right\}_{i \in I}$ be an alternate dual frame of $\left\{f_{i}\right\}_{i \in I}$. Suppose that $a$ and $b$ are real numbers such that $-1 \notin \sigma\left(S^{-a+b-1}\right)$. Then $\left\{S^{a} f_{i}+S^{b} g_{i}\right\}_{i \in I}$ is a Riesz basis for $\mathcal{H}$.

Here, we also show that the equivalence of part (1) and (2) in Proposition 3.1 of [2], is not true in general. Indeed, if $T_{1} L_{1}^{*}+T_{2} L_{2}^{*}$ is an invertible operator, then $\left\{L_{1} f_{i}+L_{2} g_{i}\right\}_{i \in I}$ is a frame for $\mathcal{H}$ but the converse is not true.

Example 2.11. Let $\left\{e_{n}\right\}_{n=1}^{\infty}$ be an orthonormal basis for $\mathcal{H}$ and $T$ be the analysis operator of $\left\{e_{n}\right\}_{n=1}^{\infty}$. Define a shift operator $L$ on $\mathcal{H}$ as in Example 2.2. Letting $L_{1}=L_{2}=L$ and $f_{n}=g_{n}=e_{n}$ for each $n \in \mathbb{N}$, in Proposition 3.1 of [2], we see that $\left\{2 L\left(e_{n}\right)\right\}_{n=1}^{\infty}$ is a frame for $\mathcal{H}$ but $2 T L^{*}$ is not a surjective operator. Indeed, $T$ is an invertible operator, but $L^{*}$ is not surjective.

Proposition 2.12. Let $\left\{f_{i}\right\}_{i \in I}$ and $\left\{g_{i}\right\}_{i \in I}$ be Bessel sequences in $\mathcal{H}$ with analysis operators $T_{1}, T_{2}$, respectively. Also, let $L_{1}, L_{2}: \mathcal{H} \longrightarrow \mathcal{H}$. Then the following are equivalent:

(1) $\left\{L_{1} f_{i}+L_{2} g_{i}\right\}_{i \in I}$ is a Riesz basis for $\mathcal{H}$.

(2) $T_{1} L_{1}^{*}+T_{2} L_{2}^{*}$ is an invertible operator on $\mathcal{H}$.

Proof. (1) $\Leftrightarrow(2)\left\{L_{1} f_{i}+L_{2} g_{i}\right\}_{i \in I}$ is a Riesz basis for $\mathcal{H}$ if and only if its analysis operator $T$ is invertible on $\mathcal{H}$ where

$$
\begin{aligned}
T f & =\left\{\left\langle f, L_{1} f_{i}+L_{2} g_{i}\right\rangle\right\}_{i \in I} \\
& =\left\{\left\langle L_{1}^{*} f, f_{i}\right\rangle+\left\langle L_{2}^{*} f, g_{i}\right\rangle\right\}_{i \in I} \\
& =T_{1} L_{1}^{*} f+T_{2} L_{2}^{*} f .
\end{aligned}
$$

\section{Applications to Gabor frames}

For $x, y \in \mathbb{R}$ we consider the operators $E_{x}$ and $T_{y}$ on $L^{2}(\mathbb{R})$ defined by $\left(E_{x} f\right)(t)=e^{2 \pi i x t} f(t)$ and $\left(T_{y} f\right)(t)=f(t-y)$. It is easy to prove that $E_{x}$ and $T_{y}$ are unitary with $E_{x}^{*}=E_{-x}$ and $T_{y}^{*}=T_{-y}$. A Gabor frame is a frame for $L^{2}(\mathbb{R})$ of the form $\left\{E_{m b} T_{n a} g\right\}_{m, n \in \mathbb{Z}}$, where $a, b>0$ and $g \in L^{2}(\mathbb{R})$ is a fixed function. We use $(g, a, b)$ to denote $\left\{E_{m b} T_{n a} g\right\}_{m, n \in \mathbb{Z}}$.

Proposition 3.1. Let $S, T \in B(\mathcal{H})$. Then $I-T S$ is surjective if and only if $I-S T$ is surjective. 
Proof. Let $I-T S$ be surjective. Then, by using Lemma 1.1 we have

$$
\begin{aligned}
(I-S T)\left(I+S(I-T S)^{\dagger} T\right) & =(I-S T)+(S-S T S)(I-T S)^{\dagger} T \\
& =I-S T+S(I-T S)(I-T S)^{\dagger} T \\
& =I-S T+S T=I,
\end{aligned}
$$

so $I-S T$ is surjective.

Corollary 3.2. Let $x, y \in \mathbb{R}$ and $c \in \mathbb{C}$ with $|c|=1$. Then the following are equivalent:

(i) $I+c T_{x} E_{y}$ is a surjective operator on $L^{2}(\mathbb{R})$.

(ii) $I+c E_{y} T_{x}$ is a surjective operator on $L^{2}(\mathbb{R})$.

Lemma 3.3. Let $T$ be a surjective and normal bounded linear operator on a Hilbert space. Then $T$ is invertible.

Proof. Assume that $T$ is not injective, i.e., there exists non-zero $x \in \operatorname{ker} T$. By normality, $\left\|T^{*} x\right\|=\|T x\|=0$. Therefore $x$ is in the orthogonal complement of the range of $T$. This implies that $T$ is not surjective.

In the following, we intend to correct Proposition 4.1 of [2].

Theorem 3.4. Let $x, y \in \mathbb{R}$ such that $x^{2}+y^{2} \neq 0$ and let $c \in \mathbb{C}$ with $|c|=1$. Then $I+c E_{y} T_{x}: L^{2}(\mathbb{R}) \longrightarrow L^{2}(\mathbb{R})$ is not surjective.

Proof. It is enough we take $x>0$. Let $f: \mathbb{R} \longrightarrow \mathbb{C}$ be a function defined by

$$
f(t):=\sum_{k=1}^{n}(-1)^{k} c^{k} \rho_{k} e^{2 \pi i k y t} \chi_{[k x,(k+1) x)}(t),
$$

where $\rho_{1}=1$ and $\rho_{k+1}=\rho_{k} e^{-2 \pi i k x y}$ for $k \geqslant 1$. By a simple computation, we get

$$
\|f\|^{2}=\int_{\mathbb{R}}|f(t)|^{2} d t=n x, \quad\left\|\left(I+c E_{y} T_{x}\right) f\right\|^{2}=2 x .
$$

Therefore $f \in L^{2}(\mathbb{R})$ and $I+c E_{y} T_{x}$ cannot be invertible. Since $E_{y}$ and $T_{x}$ are unitary, $I+c E_{y} T_{x}$ is normal. The previous lemma implies $I+c E_{y} T_{x}$ can not be surjective.

Corollary 3.5. Let $x, y \in \mathbb{R}$ such that $x^{2}+y^{2} \neq 0$ and let $c \in \mathbb{C}$ with $|c|=1$. If $(g, a, b)$ is a Gabor frame and $a y, b x \in \mathbb{Z}$, then $\left(g+c E_{y} T_{x} g, a, b\right)$ is not a Gabor frame.

Proof. It is clear that $E_{m b} T_{n a}\left(g+c E_{y} T_{x} g\right)=\left(I+d T_{x} E_{y}\right)\left(E_{m b} T_{n a} g\right)$, where $|d|=1$. If $\left(g+c E_{y} T_{x} g, a, b\right)$ is a Gabor frame, then $I+d T_{x} E_{y}$ is surjective on $L^{2}(\mathbb{R})$ by Proposition 2.1. So $I+d E_{y} T_{x}$ is surjective by Corollary 3.2. Using Theorem 3.4, we get a contradiction. 
Corollary 3.6. Let $x, y \in \mathbb{R}$ such that $x^{2}+y^{2} \neq 0$ and let $c \in \mathbb{C}$ with $|c|=1$. If $(g, a, b)$ is a Gabor frame, then the following sequence is not a Gabor frame

$$
\left\{E_{m b} T_{n a}\left(g+c e^{2 \pi i(n a y-m b x)} E_{y} T_{x} g\right)\right\}_{m, n \in \mathbb{Z}} .
$$

Proof. Because

$$
E_{m b} T_{n a}\left(g+c e^{2 \pi i(n a y-m b x)} E_{y} T_{x} g\right)=\left(I+c e^{2 \pi i x y} T_{x} E_{y}\right)\left(E_{m b} T_{n a} g\right),
$$

we get the result.

Acknowledgment. The authors would like to thank the referees for their helpful comments to improve the quality of this manuscript.

\section{References}

[1] O. Christensen, An Introduction to Frames and Riesz Bases, Birkhäuser, Boston, 2003.

[2] S. Obeidat, S. Samarah, Peter G. Casazza, and J. C. Tremain, Sums of Hilbert space frames, J. Math. Anal. Appl. 351 (2009), no. 2, 579-585.

\section{A. Najati}

Department of Mathematics

Faculty of Mathematical Sciences

University of Mohaghegh Ardabili

ARDABIL 56199-11367, IrAN

E-mail address: a.nejati@yahoo.com, a.najati@uma.ac.ir

M. R. Abdollahpour

Department of Mathematics

Faculty of Mathematical Sciences

University of Mohaghegh Ardabili

ARDABIL 56199-11367, IRAN

E-mail address: mrabdollahpour@yahoo.com, m.abdollah@uma.ac.ir

E. OSGOoeI

Department of Sciences

URmia University of TeChNOLOGy

Band High Way, Urmia, Iran

E-mail address: e.osgooei@uut.ac.ir

M. M. SAEM

Department of Mathematics

Faculty of Mathematical Sciences

University of MoHaghegh ARdabiLI

ARDABIL 56199-11367, Iran

E-mail address: m.mohammadisaem@yahoo.com 